\title{
BOUNDARY CONDITIONS IN OPTIMAL CONTROL
}

\author{
B. D. CRAVEN ${ }^{1}$
}

(Received February 1, 1988)

\begin{abstract}
Abatract
A simple rigorous approach is given to finding boundary conditions for the adjoint differential equation in an optimal control problem. The boundary conditions for a time-optimal problem are calculated from the simpler conditions for a fixed-time problem.
\end{abstract}

\section{Introduction}

When an optimal control problem is solved using the Pontryagin theory, the adjoint differential equation must be given suitable boundary conditions, depending on the terminal conditions for the given problem. Even when, in a computation, the Pontryagin maximum (or minimum) principle is not used (see e.g. [5], [6]), the adjoint equation with boundary conditions is still needed, in order to compute the gradient of the objective functional. K. L. Teo et al. [6] have obtained boundary conditions for the adjoint equation, for a class of time-optimal control problems, by a fairly complicated calculation. It will now be shown that such boundary conditions can be deduced from those for a fixed-time problem, in a manner that is simple and intuitive, as well as rigorous, and which could be extended. The approach uses Dirac delta functions, which may be regarded either as Schwartz distributions, or as functions with extended domain and range spaces [3]. While the required results for fixed-time optimal control are well known, a simple approach to them is first outlined, for the reader's convenience.

\footnotetext{
${ }^{1}$ Mathematics Department, University of Melbourne, Parkville, Vic. 3052, Australia. (C) Copyright Australian Mathematical Society 1989, Serial-fee code 0334-2700/89
} 


\section{Pontryagin theory for a fixed-time optimal control problem}

Consider the following fixed-time optimal control problem:

$$
\begin{aligned}
& \text { Minimise } F(x, u):=\int_{I} f(t, x(t), u(t)) d t \\
& \quad \text { subject to } \quad x(0)=x_{0}, \quad d x(t) / d t=m(t, x(t), u(t)(t \in I), \\
& u(t) \in \Delta(t \in I), \quad n(x(t)) \in V(t)(t \in I) .
\end{aligned}
$$

Here $I:=[0, T]$, with $T$ fixed; the functions $f(., .,$.$) and m(., .,$.$) are continuously$ differentiable; for each $t \in I, V(t)$ is a closed convex cone, representing constraint components $n_{i}(x(t)) \geq 0$ or $=0$. The differential equation may be written as $D x=M(x, u)$, where $D=d / d t$, and $M$ is a mapping of the functions $x$ and $u$, defined by $M(x, u)(t):=m(t, x(t), u(t))$. Similarly, define the mapping $N$ by $N(x)(t):=n(x(t))$. Consider the control function $u($.$) to be piecewise$ continuous, and the state function $x($.$) to be piecewise smooth, with norms$ $\|u\|:=\|u\|_{\infty}$ and $\|x\|:=\|x\|_{\infty}+\|D x\|_{\infty}$; denote these function spaces by $U$ and $X$ respectively. It then follows [2] that $F(.,$.$) and M(.,$.$) are Fréchet$ differentiable, and the linear mapping $D$ is continuous. Let $\Gamma:=\{u \in U$ : $(\forall t \in I) u(t) \in \Delta\}$. For convenience in developing the theory, assume [2] a shift of origin in $X$ to make the initial condition $x_{0}=0$; the reverse shift will be made to the results. Denote by $V(t)^{*}$ the dual cone to $V(t)$.

For problem (P1), define

$$
H(x, u):=F(x, u)+\lambda M(x, u)-\nu N(x),
$$

where $\lambda$ and $\nu$ are linear functionals in spaces dual to those in which $M(x, u)$ and $N(x)$ take their values. Then

$$
H(x, u)=\int_{I} h(t, x(t), u(t), \lambda(t), v(t)) d t
$$

where the Hamiltonian

$$
h(t, x(t), u(t), \lambda(t), v(t)):=f(t, x(t), u(t))+\lambda(t) m(t, x(t), u(t))-\nu(t) n(x(t)),
$$

and $\lambda($.$) and \nu($.$) are functions (possibly involving delta functions) which repre-$ sent the functionals $\lambda$ and $\nu$ respectively. (Thus the evaluation of the functional $\lambda$ at a function $v($.$) equals \int_{I} \lambda(t) v(t) d t$.) Let $\rho=[\lambda, \mu]$.

Assume now that the differential equation for $x(t)$, with initial condition, defines a unique $x=\Phi(u)$ for each $u \in \Gamma$, where the mapping $\Phi($.$) satisfies a$ Lipschitz condition. Let

$$
P(x, u):=[-D x+M(x, u), N(x)-s],
$$

where $s$ is a slack variable (thus, $s() \geq$.0 ). In the next calculation, assume that $x=\Phi(u)$ and $x^{\wedge}=\Phi\left(u^{\wedge}\right)$, where $u, u^{\wedge} \in \Gamma$. When there is a state constraint 
$n(x(t)) \in V(t)(t \in I)$, assume that $s($.$) is adjoined to x($.$) as additional state$ component(s). Define also $Q(.,):.=F(.,)+.\rho P(.,$.$) . Assume that F$ is partially Fréchet differentiable with respect to $x$, uniformly in $u$ near $u^{\wedge}$, thus that

$$
F(x, u)-F\left(x^{\wedge}, u\right)=F_{x}\left(x^{\wedge}, u^{\wedge}\right)\left(x-x^{\wedge}\right)+o\left(\left\|x-x^{\wedge}\right\|+\left\|u-u^{\wedge}\right\|\right),
$$

with $F_{x}$ denoting partial Fréchet derivative; and assume a similar property for $M$. (Note that usual partial Fréchet differentiability would have, instead, $F_{x}\left(x^{\wedge}, u\right)$.) Then

$$
\begin{aligned}
H\left(x^{\wedge},\right. & \left.u^{\wedge}\right)-H\left(x^{\wedge}, u\right) \\
& =F\left(x^{\wedge}, u^{\wedge}\right)-F(x, u)+F(x, u)-F\left(x^{\wedge}, u\right)+\rho\left[P\left(x^{\wedge}, u^{\wedge}\right)-P\left(x^{\wedge}, u\right)\right] \\
& =F\left(x^{\wedge}, u^{\wedge}\right)-F(x, u)+F(x, u)-F\left(x^{\wedge}, u\right)+\rho P(x, u)-\rho P\left(x^{\wedge}, u\right) \\
& =F\left(x^{\wedge}, u^{\wedge}\right)-F(x, u)+Q(x, u)-Q\left(x^{\wedge}, u\right) \\
& =F\left(x^{\wedge}, u^{\wedge}\right)-F(x, u)+Q_{x}\left(x^{\wedge}, u^{\wedge}\right)\left(x-x^{\wedge}\right)+o\left(\left\|x-x^{\wedge}\right\|+\left\|u-u^{\wedge}\right\|\right) \\
& =F\left(x^{\wedge}, u^{\wedge}\right)-F(x, u)+Q_{x}\left(x^{\wedge}, u^{\wedge}\right)\left(x-x^{\wedge}\right)+o\left(\left\|u-u^{\wedge}\right\|\right), \quad \text { (Ham) }
\end{aligned}
$$

by the assumptions of differentiability uniformly in $u$, and the Lipschitz assumption on $\Phi$.

Now, if $\left(x^{\wedge}, u^{\wedge}\right)$ minimises the control problem (P1), and if $\rho$ satisfies the equation $Q_{x}\left(x^{\wedge}, u^{\wedge}\right)=0$ (say with $\rho=\rho^{\wedge}$ ), then

$$
H\left(x, u^{\wedge}\right)-H\left(x^{\wedge}, u^{\wedge}\right) \geq o\left(\left\|u-u^{\wedge}\right\|\right) \quad\left(u \in \Gamma, u \rightarrow u^{\wedge}\right) .
$$

Thus (see [1], [2]) $H\left(., u^{\wedge}\right)$ has a quasimin at $x^{\wedge}$, subject to $u \in \Gamma$. If the minimum of the control problem is either global, or a local minimum with respect to the $L^{1}(I)$ norm for $u$, then Theorem 5.3.1 of [2] deduces from the quasimin of $H\left(., u^{\wedge}\right)$ the Pontryagin property, that

$$
(\forall w \in \Delta) h\left(t, x^{\wedge}(t), w, \lambda^{\wedge}(t)\right) \geq h\left(t, x^{\wedge}(t), u^{\wedge}(t), \rho^{\wedge}(t)\right)
$$

for almost all $t \in I$, where $\rho^{\wedge}()=.\left[\lambda^{\wedge}(),. \nu^{\wedge}().\right]$ is the function representing the functional $\boldsymbol{\rho}^{\wedge}$. Denote by $\mathrm{D}$ the differential operator $[D, 0]$ occurring in $P$, and let $\mathbf{D}^{T}$ denote its adjoint operator. Now

$$
\begin{aligned}
& H_{x}\left(x^{\wedge}, u^{\wedge}\right)=0 \Leftrightarrow(\forall z \in X) H_{x}\left(x^{\wedge}, u^{\wedge}\right) z=0 \\
& \quad \Leftrightarrow(\forall z \in X) \int_{I}\left[h_{x}(t, x(t), u(t), \lambda(t)) z(t)+\rho(t) \mathbf{D} z(t)\right] d t=0 \\
& \quad \Leftrightarrow(\forall z \in X) \int_{I}\left[h_{x}(t, x(t), u(t), \lambda(t)) z(t)-\left[\mathbf{D}^{T} \lambda(t)\right] z(t) d t+\lambda(T) z(T)=0,\right.
\end{aligned}
$$

on calculating the Fréchet derivative, and integrating by parts, using $z(0)=0$. Hence $H_{x}\left(x^{\wedge}, u^{\wedge}\right)=0$ holds exactly when $\lambda($.$) satisfies the adjoint differential$ equation:

$$
-d \rho(t) / d t=h_{x}(t, x(t), u(t), \lambda(t))(t \in I) ; \quad \rho(T)=0
$$


which expands to

$-d \lambda(t) / d t=h_{x}(t, x(t), u(t), \lambda(t))(t \in I) ; \nu(t) n(x(t))=0 ; \lambda(T)=0 ; \nu(t) \in V(t)^{*}$.

Here, the requirement $\nu(t) \in V(t)^{*}$ comes from the Karush-Kuhn-Tucker necessary conditions for the control problem. The requirement $\nu(t) n(x(t))=0$ follows from $H_{s}()=$.0 , which is part of the adjoint equation, since the adjoint of the zero differential operator is 0 . Then, whenever component $n_{i}(x(t))>0, H_{s}()=$. requires $\nu_{i}(t)=0$.

Now suppose instead that the control problem (P1) includes also a parameter $p$; denote the optimal objective value now also by $J(p)$. In (Ham), suppose now that $(x, u)$ correspond to parameter value $p$, and $\left(x^{\wedge}, u^{\wedge}\right)$ correspond to parameter value $p^{\wedge}$. If also $(x, u)$ is a Lipschitz function of $p$, then (Ham) shows that

$$
F(x, u)-F\left(x^{\wedge}, u^{\wedge}\right)=H\left(x^{\wedge}, u\right)-H\left(x^{\wedge}, u^{\wedge}\right)-o\left(\left\|p-p^{\wedge}\right\|\right),
$$

assuming that $\boldsymbol{\rho}=\boldsymbol{\rho}^{\wedge}$ satisfies the adjoint differential equation. Hence, showing now the explicit dependence of $h($ ) on $p$, arising from the explicit dependence of $f$ and $m$ on $p$, there follows

$$
\begin{aligned}
J(p)-J\left(p^{\wedge}\right)= & \int_{I}\left[h\left(t, x^{\wedge}(t), \rho^{\wedge}(t), p\right)-h\left(t, x^{\wedge}(t), u^{\wedge}(t), \rho^{\wedge}(t), p\right)\right] d t \\
& +o\left(\left\|p-p^{\wedge}\right\|\right) \\
= & {\left[\int_{I} h_{p}\left(t, x^{\wedge}(t), u(t), \rho^{\wedge}(t), p^{\wedge}\right) d t\right]\left(p-p^{\wedge}\right)+o\left(\left\|p-p^{\wedge}\right\|\right) . }
\end{aligned}
$$

Hence the Fréchet derivative $J^{\prime}\left(p^{\wedge}\right)$ is given by

$$
J^{\prime}\left(p^{\wedge}\right)=\int_{I} h_{p}\left(t, x^{\wedge}(t), u(t), \lambda^{\wedge}(t), p^{\wedge}\right) d t .
$$

If, instead of introducing a parameter $p$, the gradient is sought of the objective function $\mathbf{J}(u):=F(\Phi(u), u)$, then a similar calculation gives that

$$
\mathbf{J}(u)-\mathbf{J}\left(u^{\wedge}\right)=\int_{I} h_{u}\left(t, x^{\wedge}(t), u(t), \rho^{\wedge}(t), p^{\wedge}\right)\left[u(t)-u^{\wedge}(t)\right] d t+o\left(\left\|u-u^{\wedge}\right\|\right)
$$

So the gradient of $\mathbf{J}($.$) is thus calculable.$

\section{Boundary conditions for the time-optimal control problem}

Consider now the time-optimal control problem:

$$
\begin{gathered}
\text { Minimise } J=R\left(x\left(t^{*}\right)\right)+\int_{0}^{t^{*}} f(t, x(t), u(t)) d t \text { subject to } \\
x(0)=x_{0}, \quad d x(t) / d t=m(t, x(t), u(t)) \quad\left(0 \leq t \leq t^{*}\right) ; \\
u(t) \in \Delta \quad\left(0 \leq t \leq t^{*}\right) ; \quad q\left(x\left(t^{*}\right)\right)=0 .
\end{gathered}
$$


Note that, in this problem, all of $u(),. x($.$) , and t^{*}$ are varied to reach the optimum; $t^{*}$ denotes the (variable) time to optimality. The function $q$, describing the terminal constraint, is here real valued. For brevity, ${ }^{*}$ will be used to label the values of functions evaluated at $t=t^{*}$.

Now $J$ can be rewritten as

$$
J=\int_{I}\left[f(t, x(t) \cdot u(t)) \pi\left(t^{*}-t\right)+R(x(t)) \delta\left(t-t^{*}\right)\right] d t
$$

where $I=[0, K\}$, for any fixed $K>t^{*}$, and $\pi(\tau)=0(\tau<0), \pi(\tau)=1(\tau>0)$; $\delta($.$) is Dirac's delta function. For the resulting fixed-time optimal control problem,$ the Hamiltonian is

$$
\begin{aligned}
h\left(t, x(t), u(t), \lambda(t), t^{*}\right)=f(t, x(t), u(t)) \pi\left(t^{*}-t\right)+R(x(t)) \delta\left(t-t^{*}\right) \\
\\
+\lambda(t) m(t, x(t), u(t))-\beta \delta\left(t-t^{*}\right) q(x(t)) .
\end{aligned}
$$

Here, $\lambda(t)$ is the costate (= Lagrange multiplier) function. The state constraint $q\left(x\left(t^{*}\right)\right)=0$ has been rewritten as $(\forall t) q(x(t)) \in V(t)$, where the cone $V(t)=\mathbf{R}$ except at $t=t^{*}$, where $V\left(t^{*}\right)=\{0\}$. So the corresponding costate function $\nu(t)=0$ except when $t=t^{*}$, so that $\nu(t)=-\beta \delta\left(t-t^{*}\right)$, for some constant $\beta$, may be appropriately assumed, subject to verification that this solution satisfies all necessary conditions.

The adjoint differential equation is then

$$
\begin{aligned}
-d \lambda(t) / d t= & f_{x}(t, x(t), u(t)) \pi\left(t^{*}-t\right)+\lambda(t) m_{x}(t, x(t), u(t))-\beta \delta\left(t-t^{*}\right) q_{x}(x(t)) \\
& +R_{x}(x(t)) \delta\left(t-t^{*}\right),
\end{aligned}
$$

with boundary condition $\lambda(K)=0$. Without loss of generality, $K=t^{*}+0$ may be assumed, in deriving necessary conditions. Integrating the adjoint differential equation from $t=t^{*}-0$ to $t=t^{*}+0$, only the delta function terms contribute, and then there follows

$$
\lambda\left(t^{*}-0\right)=R_{x}\left(x\left(t^{*}\right)\right)-\beta q_{x}\left(x\left(t^{*}\right)\right) .
$$

which may be briefly written as $\lambda^{*} \equiv \lambda\left(t^{*}-0\right)=R_{x}^{*}-\beta q_{x}^{*}$.

So far, the optimality of $t^{*}$ has not been used in the calculation. To do this, $t^{*}$ may be regarded as a parameter. For optimality, the derivative of $J$ with respect to $t^{*}$ should be equated to zero. From (GRAD) above, this gradient equals the integral over $I$ of $\partial h / \partial t^{*}$, where $h$ is the Hamiltonian. Now (suppressing some obvious arguments),

$$
\partial h / \partial t^{*}=f \cdot \delta\left(t^{*}-t\right)+\beta \delta^{\prime}\left(t-t^{*}\right) \cdot q-R(x(t)) \delta^{\prime}\left(t-t^{*}\right) .
$$

Integrating with respect to $t$ over $I$, and also integrating the $\delta^{\prime}$ terms by parts, the requirement is that

$$
0=f^{*}+R_{x}^{*} m^{*}-\beta q_{x}^{*} m^{*}
$$


noting that

$$
(\partial / \partial t) R(x(t))=R_{x}(x(t)) \cdot(d / d t) x(t)=R_{x}(x(t)) \cdot m(t, x(t), u(t)) .
$$

Thus the optimal value of $\beta$ is determined. Substituting it into $\left(^{*}\right)$ yields the following boundary condition for the adjoint differential equation:

$$
\lambda^{*}=R_{x}^{*}-\left[f^{*}+R_{x}^{*} m^{*}\right] q_{x}^{*} /\left[q_{x}^{*} m^{*}\right] .
$$

The formula (BC) was first obtained by K. L. Teo et al [6], by a complicated calculation of some four pages, using the chain rule. It is noted that this formula is essential for numerically computing a time-optimal control problem, since the costate function $\lambda^{\wedge}(t)$ is needed in order to obtain the gradient of the objective function with respect to $u$, allowing for the dependence of $x$ on $u$.

\section{An example}

The following example of a time-optimal control problem is given, without solution, in [4], p. 57. A vehicle moves in a plane with velocity of magnitude 1. Its Cartesian coordinates $x_{1}(t)$ and $x_{2}(t)$ in the plane, and its direction $x_{3}(t)$, are described by the differential equations

$$
d x_{1}(t) / d t=\cos x_{3}(t), \quad d x_{2}(t) / d t=\sin x_{3}(t), \quad d x_{3}(t) / d t=u(t),
$$

where the control function $u(t)$ is bounded by $(\forall t)|u(t)| \leq 1$. The vehicle is to be driven from $\left(x_{1}(0), x_{2}(0), x_{3}(0)\right)=(4,0, \pi / 2)$ to $\left(x_{1}\left(t^{*}\right), x_{2}\left(t^{*}\right)\right)=(0,0), x_{3}\left(t^{*}\right)$ not specified, in minimum time $t^{*}$. In the objective function, $f(., .,.) \equiv 1$, and $R\left(x\left(t^{*}\right)\right)=0$. An optimum solution has a switching time at $t=t_{1}$, and a singular arc for $t \in\left(t_{1}, t^{*}\right)$, and takes the form:

$$
\begin{array}{ll}
\left(0<t<t_{1}\right) & u(t)=1, \quad x_{1}(t)=3+\cos t, \quad x_{2}(t)=\sin t, \quad x_{3}(t)=\pi / 2+t, \\
& \lambda(t) \equiv\left[\lambda_{1}(t), \lambda_{2}(t), \lambda_{3}(t)\right]=(c \cos \beta, c \sin \beta,-c+c \sin (t-\beta)) \\
\left(t_{1}<t<t^{*}\right) & u(t)=0, \quad x_{1}(t)=\left(t^{*}-t\right) \cos \beta, \quad x_{2}(t)=\left(t^{*}-t\right) \sin \beta, \\
& x_{3}(t)=\pi / 2+t_{1}, \lambda(t) \equiv\left[\lambda_{1}(t), \lambda_{2}(t), \lambda_{3}(t)\right]=(c \cos \beta, c \sin \beta, 0) .
\end{array}
$$

Here $c$ is a positive constant, and $\beta=t_{1}-\pi / 2$, with $t_{1} \approx 1.9806$ and $t^{*} \approx 4.7386$. The Pontryagin theory requires $\lambda_{3}(t)<0$ when $u(t)=1$, which holds since $-1+\sin \left(t-\left(t_{1}-\pi / 2\right)\right)<0$ for $0<t<t_{1}$, and $=0$ at $t=t_{1}$, also $\lambda_{3}(t)=0$ for $t_{1}<t<t^{*}$. Since this problem is symmetric in $\operatorname{sgn} x_{2}(t)$, there is another optimal solution with the opposite sign of $x_{2}(t)$. Any positive constant $c$ will do here.

Consider now the boundary condition formula (BC), taking

$$
q(x(t)):=\sqrt{\left(x_{1}(t)^{2}+x_{2}(t)^{2}\right)}
$$


For $t^{*}-t$ small and positive, $x_{2}(t) \approx-\left(t-t^{*}\right) \cos \beta$ and $x_{2}(t) \approx-\left(t-t^{*}\right) \sin \beta$, where $x_{3}\left(t^{*}\right)=\pi+\beta$. (These approximations represent an informal use of l'Hopital's rule.) Hence

$q_{x}^{*} \approx\left[\left(t^{*}-t\right) \cos \beta,\left(t^{*}-t\right) \sin \beta, 0\right] /\left(\left(t^{*}-t\right)\left(\cos ^{2} \beta+\sin ^{2} \beta\right)\right)=[\cos \beta, \sin \beta, 0]$.

Then $q_{x}^{*} m^{*} \approx[\cos \beta, \sin \beta, 0]^{T}[-\cos \beta,-\sin \beta, 0]=-1$. From (BC),

$$
\begin{aligned}
\lambda^{*} & =0-(1+0)[\cos \beta, \sin \beta, 0] /(-1)=[\cos \beta, \sin \beta, 0] \\
& =\left[-\cos x_{3}\left(t^{*}\right),-\sin x_{3}\left(t^{*}\right), 0\right] .
\end{aligned}
$$

If instead $q(x(t))=(1 / 2)\left[x_{1}(t)^{2}+x_{2}(t)^{2}\right]$ then, since $x_{1}\left(t^{*}\right)=0$ and $x_{2}\left(t^{*}\right)=$ 0 , the limiting case $q_{x}^{*}=0, \lambda^{*}=0$ arises. Although this choice of $q(x(t))$ specifies the same terminal point to the path as does the previous $q(x(t))$, they do not appear to be computationally equivalent.

\section{References}

[1] B. D. Craven, "Lagrangian conditions and quasiduality", Bull. Austral. Math. Soc. 16 (1977), 325-339.

[2] B. D. Craven, Mathematical Programming and Control theory, (Chapman \& Hall, London, 1978).

[3] B. D. Craven, "Generalised functions for applications", J. Austral. Math. Soc., Ser. B, 28 (1985), 362-374.

[4] W. H. Fleming and R. W. Rishel, Deterministic and Stochastic Optimal Control, (SpringerVerlag, Berlin, 1975).

[5] C. J. Goh and K. L. Teo, "MISER, an Optimal Control Software", Dept. of Industrial \& Systems Engineernng, National University of Singapore, Singapore, 1987.

[6] K. L. Teo, G. Jepps, E. J. Moore and S. Hayes, "A computational method for free time optimal control problems, with application to maximising the range of an aircraft-like projectile", J. Austral. Math. Soc., Ser. B, 28 (1987), 393-413. 